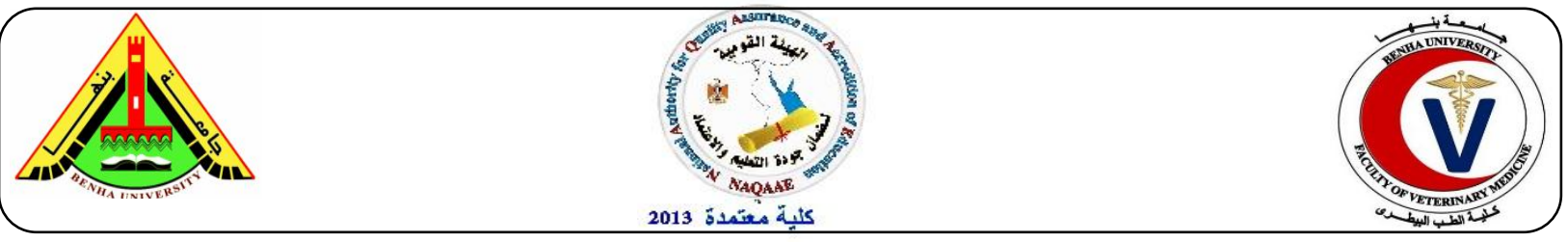

\title{
Effect of Nanoselenium on Experimentally Induced Mammary Cancer in female Rats: Possible Role of TGF- $\beta 1$
}

\author{
Omayma, A.R. Abou-Zaid ${ }^{1}$, Laila A Rashed ${ }^{2}$, El-Sonbaty, S. M. ${ }^{3}$ and Aboel-Ftouh, A. I. ${ }^{4}$ \\ ${ }^{1}$ Head of Clinical Biochemistry, Faculty of Veterinary Medicine, Benha University, ${ }^{2}$ Department of \\ Biochemistry and Molecular Biology, Faculty of Medicine, Cairo University, Cairo, Egypt, ${ }^{3}$ Assistant \\ Professor Microbiology department, National center for Radiation Research and Technology,Atomic Energy \\ Authority, Cairo, Egypt, ${ }^{4}$ Biochemistry department, Faculty of Veterinary Medicine, Benha University
}

\section{A B S T R A C T}

Nanoparticles combined with natural products could be an efficient tool in cell base cancer gene therapy. Guided treatments with nanoparticles and radiotherapy are a new approach in cancer therapy. The current study evaluated the beneficial antitumor effects of SeNPs, $\gamma$-radiation together with selenium nanoparticles (SeNPs) against mammary gland cancer in female Rats.

(http://www.bvmj.bu.edu.eg)

(bvmj, 35(1): 362-368, March, 2018)

\section{INTRODUCTION:}

Breast Cancer (BC) is the most common neoplasia among women and has high incidence and mortality rates worldwide (Torre et al., 2015). National Cancer Institute (Instituto Nacional de Câncer, INCA) has estimated 57,960 new BC cases for 2016, categorizing this disease as a public health burden. Breast Cancer is considered to be a heterogeneous disease with different gene expression profiles that translate into various clinical outcomes such as survival rate, disease relapse, site of preference of metastatic spread and chemotherapy response. Breast Cancer molecular stratification is currently based on hormonal receptor expression (estrogen, progesterone and Human epidermal receptor 2 HER2), with 4 major molecular subtypes: Luminal, Luminal- HER2, HER2 and Triple negative (TN). Additional factors such as age, tumor grade (I, II, III, or IV), genetic stability, hormone therapy status (anti-conception therapy and post-menopausal hormone therapy), tumor localization (e.g., ductal carcinoma, metastasis), particular protein expression and, most importantly, early diagnosis may correlate with BC development, prognosis, tumor progression, and treatment success (Cornejo-Moreno et al., 2014). Although extensive investigation into understanding $\mathrm{BC}$ has led to substantial progress in disease outcomes, many other factors are yet to be clarified.

Therefore, the need for non-invasive approaches in cancer is extremely important for prognosis, especially in subtypes with a poor prognosis. Within this context, several bio fluids have been investigated to identify biomarkers in cancer that could improve therapy with regard to several aspects, such as disease diagnosis, classification, treatment/ 
therapy follow-up, relapse and resistance. Plasma represents a "perfect" non-invasive source for molecular investigation. Nevertheless, due to its complexity, plasma has not been well explored in the search for markers of $\mathrm{BC}$ when compared with proteomic studies utilizing tissue samples or cell line secretes (Zhang et al., 2013).

In nanotechnology, a particle is defined as a small object with size ranges between 1 and 100 that behaves as a whole unit in terms of its transport and properties. NP's are mainly used in the drug delivery system as their particle size and surface characteristics can be easily manipulated to achieve both passive and active drug targeting. Further, they can be used to provide control and sustain release of the drug during the transportation at the site of action. They alter organ distribution of the drug and subsequent clearance of the drug so as to achieve increase in drug therapeutic efficacy and reduction in side effects (Zhang et al., 2013).

Selenium is one of the essential trace elements in the body in due to its antioxidative as well as pro-oxidative effect and has great importance in nourishment and medicine (Zhang et al., 2014). Selenium is a key player in cellular metabolism, an essential component of enzymes that protect the body against free radical species and has important roles in metabolism of thyroid, human fertility and many other vital functions. All aspects of Se in biology have advanced in various fields such as genetic, biochemical, molecular, and health areas. Many stable organic selenium compounds have been successfully synthesized which are used as antioxidants, enzyme inhibitors, anti-tumor, anti-infective agents, cytokine inducers and immunomodulators (Sies and Masumoto., 1997). Nanoparticles of selenium act as a potential chemo-preventive agent with reduced toxicity
(Wang et al., 2007). For example it has been reported that the redness selenium nanoparticles has high biological activities and low toxicity. Thus selenium nanoparticles caused the great interest of researchers and a variety of synthesis methods have been exploited (Hassan et al., 2014).

The complete understanding of the synthesis mechanism of nanoparticles using the biological agents has not been devised. The biological synthesis mechanism includes both intra and extracellular of nanoparticles which are different for various biological agents and different biomolecules responsible for the synthesis of these nanoparticles. Biological agents used for nanoparticles synthesis represent mainly microbes including bacteria, fungi, algae and yeast and plants which react differently with metal ions (Hassan et al., 2014).

Biochemicals can be used for the synthesis of nanomaterials, however the biogenic synthetic route is frequently used due to its ease and simplicity. In addition, there are no hazardous and toxic residues released in the environment (Sharma et al., 2014). It has been established that the Se nanoparticles prepared from biological material are less toxic than the bulk Se nanoparticles prepared from chemicals. The biomolecules present in the extract act both as reducing agent and stabilizers of $\mathrm{Se}$ nanoparticles. Green synthesis of selenium nanoparticles from selenious acid was achieved by dried extract of raisin (Vitis vinifera) (Li et al., 2007).

This red color is the characteristic indication of $\mathrm{Se}$ nanoparticles. The Se nanoparticles synthesized from fenugreek seed extract in aqueous medium at room temperature are between $50-150 \mathrm{~nm}$ and have been found to be active against human breast cancer cells (Ramamurthy et 
al.,2013).Accordingly, The aim of this study to investigate the effect of SeNPs as breast cancer inhibitor through TGF- $\beta$ modulation.

\section{MATERIALS AND METHODS:}

- Soy milk was provided by Microbio Soy factor, Food Technology Institute Agricultural research center, Giza, Egypt.

- Selenium dioxide $\left(\mathrm{SeO}_{2}\right.$, molecular weight: 110.96) Sigma-Aldrich, part of Merck Company, USA.

- 7,12- di- methyl benz (a) anthracene (DMBA) (Sigma USA) Company.

- Estradiol accelerate the development of 7,12 dimethyl benz[a] anthracene (DMBA)-induced mammary tumors.

\section{Preparation of 7,12-di-methyl benz (a)}

\section{anthracene (DMBA):}

1) 7,12- di- methyl benz (a) anthracene (DMBA) was dissolved in sesame oil in such a way that each $\mathrm{ml}$ was containing $5 \mathrm{mg}$ of DMBA and was administered at the dose rat of $50 \mathrm{mg}$ $/ \mathrm{kg}$ body weight intra-peritoneal (i.p). (Fisher et al., 1992).

Preparation of Fermented soy milk (FSM):

Fermented soy milk (FSM) was prepared according to (Chung et al.,2002). The microorganisms used in the fermenting process included Lactobacillus acidophilus, Lactobacillus bulgaricus, Streptococcus lactis, Bifidobacteria, and yeasts, which are found as intestinal microflora and in some traditional fermented products.

\section{Selenium nanoparticles preparation (SeNPs)}

The aqueous part of fermented soy obtained was used as a pre-cursor for synthesis of SeNPs. The aqueous part of fermented soy $(2 \mathrm{ml})$ was added drop wise into the $20 \mathrm{ml}$ solution of $\mathrm{SeO}_{2}(10 \mathrm{mM})$, with vigorous stirring. The mixture was incubated by placing the solution onto a rotatory orbital shaker operating at $200 \mathrm{rpm}, 30{ }^{\circ} \mathrm{C}$ for $72 \mathrm{~h}$ in dark conditions. The reduction of selenium ions was monitored by sampling an aliquot $(3 \mathrm{ml})$ of the mixture at intervals of $24 \mathrm{~h}$, followed by measurement of absorption maximum. Absorption maximum was determined by measuring optical density of the content from wavelength 350 to $700 \mathrm{~nm}$ using UV-Vis spectrophotometer. (Berhanu et al.,2009).

\section{Se Nanoparticles Characterization:}

An initial characterization of the test substance is imperative before any toxicity screening is commenced. A more extensive and complete characterization, including size distribution, shape, solubility, etc., is recommended for nanomaterials in order to determine the correct correlation between their physicochemical properties and the biological effects they elicit (Berhanu et al., 2009).

Determination of LD50 using experimental animals. In screening drugs, determination of LD50 is usually an initial step in the assessment and evaluation of the toxic characteristics of a substance. The LD50 of the SeNPs was determined as described by (Akhila et al ., 2007).

\section{Experimental animals:}

Forty virgin female Sprague-Dawley of 4 weeks old, with body weight range of 80$100 \mathrm{~g}$ were used in the present work. Rats were purchased from the Egyptian Holding Company for Biological Products and Vaccines (Cairo, Egypt). All animals were fed a standard diet in the form of cubes containing $20 \%$ casein, $13 \%$ sucrose, $50 \%$ corn starch, $10 \%$ corn oil, $2 \%$ vitamins mixture and $0.5 \%$ $\mathrm{NaCl}$ and had access of tap water all the time.

Experimental design: 
The present study was carried out on forty virgin female rats DMBA was administrated as 50mg DMBA/kg body weight (Fisher et al., 1992).

At the beginning of the experiment rats were divided into 4 main groups:

Group (1) control: Rats served as negative control and orally received saline.

Group (2) DMBA: Rats rats were injected i.p. with DMBA (50 mg/kg).

Group (3) SeNPs: Rats were orally administrated with $10 \%$ of $\mathrm{LD}_{50}$ of SeNPs (2 $\mathrm{ml}, 20 \mathrm{mg} / \mathrm{kg}$ body weight) daily.

Group (4) DMBA + SeNPs: Rats were injected with DMBA i.p. then orally administrated with SeNPs $(2 \mathrm{ml}, 20 \mathrm{mg} / \mathrm{kg}$ body weight).

\section{Sampling:}

At the end of the treatment period, animals were fasted overnight prior to dissection under light ether anesthesia. Blood was drawn from the vena cava and centrifuged at $3000 \mathrm{rp} . \mathrm{m}$ for $10 \mathrm{~min}$.

\section{Experimental parameters:}

All Serum were analyzed to determine of TGF- $\beta$ according to catalog no. MBS728582.using rat TGF- $\beta$ sandwich ELISA kit purchased from MyBioSource, Inc. (USA).

Statistical analysis.:

All mean values are reported as the mean \pm standard deviation (SD). Data were analyzed using a one-way analysis of variance (ANOVA). The level of significance between mean values was set at $p<0.05$ and $p<0.01$ (significant and highly significant, respectively). All statistical analyses were performed using SPSS software (version 20.0).

Chemical and biological parameters:

Assessment of TGF- $\beta$ level by ELISA.

Analysis using elissa technique revealed significant elevated level of TGF- $\beta$ of female rate treated with DMBA. SeNPs,

\section{RESULTS:}

Table (1): The effect of Selenium nanoparticles on Transforming growth factor- $\beta$ (TGF- $\beta$ ) levels in plasma of female rats induced mammary gland carcinoma.

\begin{tabular}{|c|c|}
\hline Groups & $\begin{array}{c}\text { Transforming growth factor- } \beta \text { TGF- } \beta \\
(\mathrm{pg} / \mathrm{mg})\end{array}$ \\
\hline Control & $33.08 \pm 0.98^{\mathrm{b}}$ \\
\hline DMBA & $175.35 \pm 3.06^{\mathrm{a}}$ \\
\hline SNPs & $24.33 \pm 1.13^{\mathrm{ab}}$ \\
\hline DMBA + SNPs & $75.18 \pm 2.07^{\mathrm{ab}}$ \\
\hline
\end{tabular}

Values are expressed as means \pm Standard Errors $(n=6)$ where a value considered significant different at $\mathrm{p}<0.05$. 


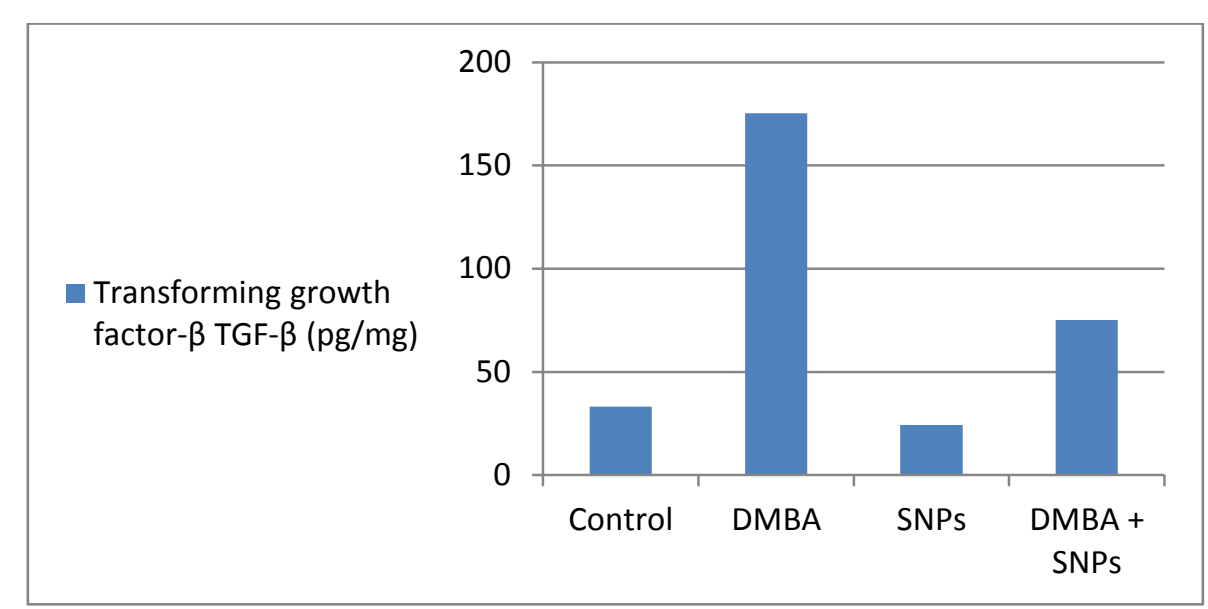

Fig (1) :Transforming growth factor- $\beta$ TGF- $\beta$ (pg/mg)

\section{DISCUSSION:}

Breast cancer (BC) was the most common malignant tumor in the world and it was also an important cause of death in women. Its occurrence and development were a complex process involving many factors, including the activation of oncogenes and inactivation of tumor suppressor genes, and finally induced the changes of cell signaling pathway, cell proliferation, apoptosis, in vision and so on.

\section{Selenium nanoparticles (SeNPs)} synthesized using soy milk showed antitumor activity via inhibition of TGF- $\beta$ oncogenic signaling pathway and stimulate suppression pathway. Soy milk was reported decreased TGF- $\beta 1$ production by Nano differentiated monocytic U937 cells. Different suppression patterns was reported of soy milk and ferment soy milk. This suggests the influence of probiotic by-products results from fermentation process. Also the presence of isoflavones, such as genistein and daidzein, in soy milk gives the probiotic bacteria a unique substrate for fermentation (Yeo and Liong., 2010).
The current study discovered the changes in level in TGF- $\beta$ in serum of female rate when they were administrated with SeNPs has significant carcinoma cell growth suppressing abilities via suppressing signals gene like (TGf- $\beta$ ). TGF- $\beta$ 's complicated biological responses have been proposed to be governed by the different cellular contextual determinants of Smads, including a wideranging complement of DNA-binding transcription factors. (Massague et al., 2012)

TGF- $\beta$ signaling also promotes cancer metastasis through upregulation of EMT regulatory factors, Despite the variation in the rate of genetic mutations in TGF- $\beta \mathrm{R}$ and Smad components, most epithelial-derived tumors including pancreatic, colon, breast and ovarian cancers finally become resistant to the growth-inhibitory effects of TGF- $\beta$. whereas in tumors including breast and ovarian cancers, the defection of DPC4/Smad4 was only detected in one-eighth of cancer samples and the mechanism of resistance was more complicated, including the inability of TGFBR proteins, overexpression of inhibitory Smads, and suppression of canonical TGF- $\beta$ signaling 
by other oncoproteins such as Ras and Myc. (Elliott and Blobe., 2005).

The increased expression of TGF- $\beta$ in tumor microenvironment could also facilitate tumor progression through interacting with fibroblast, endothelial cells or infiltrating immune cells. TGF- $\beta$ is able to directly induce fibroblasts to myofibroblasts with characteristic morphological changes and upregulation of a-SMA, which play a crucial role in supporting the growth of tumor cells. Many studies implicated the angiogenesis-inducing capabilities of TGF- $\beta$ in accelerating tumor progression TGF- $\beta 1$ expression by tumor or stroma cells may also show immunosuppressive effect, through impairing the function of both CD4+ and CD8+ T cells as well as natural killer cells (Jakowlew., 2006).

\section{CONCLUSION:}

In conclusion our results suggest that selenium nanoparticles companied with fermented soy milk can be used as treatment of breast cancer and effect on (TGF- $\beta$ ) leading to inhibition of tumour growth.

\section{REFERENCES :}

Akhila, JS, Deepa S and Alwar MC. Acute toxicity studies and determination of median lethal dose. Curr Sci 2007; 93: 917-920

Berhanu D, Dybowska A, Misra SK, Stanley CJ, Ruenraroengsak P, et al. (2009) Characterisation of carbon nanotubes in the context of toxicity studies. Environ Health 8 Suppl 1: S3.

Chung, Y.C., Chang C.T., Chao W.W., Lin C.F., Chou S.T. (2002): Antioxidative activity and safety of the 50 ethanolic extract from red bean fermented by Bacillus subtilis IMR-NK1. Journal of
Agricultural and Food Chemistry 50:2454-2458.

Cornejo-Moreno, B.A., D. Uribe-Escamilla, F. Salamanca-Gómez, Breast cancer genes: looking for BRACA's lost brother, Isr. Med. Assoc. J. 16 (2014) 787-792.

Elliott R.L., G.C. Blobe, Role of transforming growth factor beta in human cancer, J. Clin. Oncol. 23 (2005) 2078-2093.

Fisher,S.M., Conti,C.J., Locniskar,M., Belury,M.A., Maaldve,R.E., Lee,M.L., Leyton,J., Slaga,T.J. and Bechtel,D.H. (1992) The effect of dietary fat on the rapid development of mammary tumors induced by 7,12dimethylbenz[a]anthracene in SENCAR mice. Cancer Res., 52,662-666.

Gurunathan S., et al. / Colloids and Surfaces B: Biointerfaces 74 (2009) 328-335

Hassan R El-Ramady, Éva DomokosSzabolcsy, Neama AA, Tarek AA, Tarek AS, et al. (2014) Selenium and nanoselenium in agro ecosystems. Environ Chem Lett 12: 495.

Jakowlew S.B., Transforming growth factorbeta in cancer and metastasis, Cancer Metastasis Rev. 25 (2006) 435-457.

Li SK, Shen YH, Xie AJ, Yu XR, Zhang XZ, et al. (2007) Rapid, room-temperature synthesis of amorphous selenium/protein composites using Capsicum annuum L. extract. Nanotechno 18: 405101-405109.

Massague J. TGFbeta signalling in context. Nat Rev Mol Cell Biol2012;13(10):61630

Montes-Burgos I, Walczyk D, Hole P, Smith J, Lynch I, et al. (2010) Characterization of nanoparticle size and state priorto nanotoxicological studies. J Nanopart Res 12: 47-53.

Ramamurthy CH, Sampath KS, Arunkumar P, Kumar MS, Sujatha V, et al. (2013) Green synthesis and characterization of selenium nanoparticles and its 
augmented cytotoxicity with doxorubicin on cancer cells. Bioprocess Biosyst Eng 36: 1131.

Sharma G, Sharma AR, Bhavesh R, Park J, Ganbold B, et al. (2014) Biomoleculemediated synthesis of selenium nanoparticles using dried Vitis vinifera (raisin) extract. Molecules 19: 27612770.

Sharma G, Sharma AR, Bhavesh R, Park J, Ganbold B, et al. (2014) Biomoleculemediated synthesis of selenium nanoparticles using dried Vitis vinifera (raisin) extract. Molecules 19: 27612770.

Sies H, Masumoto H (1997) Ebselen as a glutathione peroxidase mimic and as a scavenger of peroxynitrite. Adv Pharmacol 38: 229-246.

Torre L.A., F. Bray, R.L. Siegel, J. Ferlay, J. Lortet-Tieulent, A. Jemal, Global cancer statistics, 2012, CA Cancer J. Clin. 65 (2015) 87-108.

Wang H, Zhang J, Yu H (2007) Elemental selenium at nano size possesses lower toxicity without compromising the fundamental effect on selenoenzymes: Comparison with selenomethionine in mice. Free Radic Biol Med 42: 15241533

Xiao F, Zhao F, Mei D, Mo Z, Zeng B (2009) Non-enzymatic glucose sensor based on ultrasonic-electrodeposition of bimetallic $\mathrm{PtM}(\mathrm{M}=\mathrm{Ru}, \mathrm{Pd}$ and $\mathrm{Au})$ nanoparticles on carbon nanotubes-ionic liquid composite film. Biosens Bioelectron 24: 3481-3486.

Yeo, S. K., \& Liong, M. T. (2010). Angiotensin I-converting enzyme inhibitory activityand bioconversion of isoflavones by probiotics in soymilk supplemented with prebiotics.
International Journal of Food Science and Nutrition, 61(2), 161-181.

Zhang J, Zhang SY, Xu J, Chen HY (2004) A new method for the synthesis of selenium nanoparticles and the application to construction of $\mathrm{H} 2 \mathrm{O} 2$ biosensor. Chin Chem Lett 15: 13451348.

Zhang, F, M. Wang, T. Michael, R. Drabier, Novel alternative splicing isoform biomarkers identification from highthroughput plasma proteomics profiling of breast cancer, BMC Syst. Biol. (7 Suppl 5) (2013) S8. 